\title{
Relations between the Experience of the Yips and Athletes' Psychological Growth
}

\author{
Kojiro Matsuda ${ }^{*}$, Yasuo Susaki ${ }^{2}$, Yoshio Sugiyama ${ }^{3}$ \\ ${ }^{1}$ Graduate School of Human-Environment Studies, Kyushu University, 6-1 Kasuga-koen, Kasuga-City, Fukuoka, Japan \\ ${ }^{2}$ Faculty of Education, ShimaneUniversity, 1060, Nishikawatsu, Matsue, Shimane 690-8504 \\ ${ }^{3}$ Factory of Human-Environment Studies, Kyushu University, 6-1 Kasuga-koen, Kasuga-City, Fukuoka, Japan
}

Received: March 09, 2018; Accepted: May 09 2018; Published: May 162018

*Corresponding author: Kojiro Matsuda, Graduate School of Human-Environment Studies, Kyushu University, 6-1 Kasuga-koen, Kasuga-City, Fukuoka, Japan Tel: 81-80-8890-8773, E-mail:matsuda-k@students.ihs.kyushu-u.ac.jp

\begin{abstract}
The present study aimed to examine and clarify the relations between the experience of the 'yips' or overcoming the yips and athletes' psychological growth. A questionnaire survey was distributed and responses from 416 members $(M=19.6, \mathrm{SD}=1.1$ years) of university regulation baseball clubs were analyzed. The 416 baseball players were divided into three groups: the non-yips group, yips group, and those that overcame the yips. A one-way analysis of variance was performed, setting group as the independent variable and the five subscale scores on the Psychological Maturity as an Athlete Scale as the dependent variables. The group that overcame the yips showed significantly higher subscale scores than did the nonyips group in clear meaning of sports activity, self-understanding, and independent achievement orientation. Contrastively, there were no subscales on which the yips group had significantly higher scores than the non-yips group. The present findings suggest that the psychological development observed in people who have experienced the yips is not merely because of the experience of the yips, as overcoming the yips also plays an important role in this development.
\end{abstract}

Keywords: Posttraumatic growth (PTG); stress-related growth (SRG); negative experiences; crisis; turning point

\section{Introduction}

Traditionally, the 'yips' has been known as a movement disorder observed when a golfer is putting (e.g., [1]) and according to a survey that McDaniel, et al. [1] conducted on 1050 golfers, there was a $42 \%$ response rate and $28 \%$ reported that they had experienced the yips. Furthermore, according to a survey that Smith, et al. [2] conducted on 1031 golfers, 541 (52\%) responded that they had experienced the yips in the past. On the other hand, baseball players also suffer from the yips in the same way $[3,4]$. Commonly noted characteristics of the yips include a sudden inability to perform a previously natural action or motion [2], an inability to control one's own body as intended [5], and chronic or perpetual symptoms [6, 7]. As reported in baseball, the yips primarily manifests in actions like pitching and throwing the ball [8]. Therefore, previous studies on the yips in baseball players have used special terminology such as "psychogenetic loss of pitching motions" [9], "loss of pitching" [10], and "pitching and throwing disability" [11]. Additionally, in baseball, the yips is defined as "throwing or pitching wildly either up and down or left and right and having this state continue for at least one month" [12]. Thus, as per this explanation, the yips is defined as a movement disorder observed when someone throws or pitches a ball.

In examining previous studies on the yips, it becomes apparent that most studies have focused on the factors causing the onset of the yips and the coping methods for dealing with these factors. The factors that could cause the onset of the yips are mainly neurological factors such as task-specific focal dystonia [13] and psychological factors such as reinvestment (e.g., [14]). Additionally, the coping methods used to treat the neurological aspects of the yips that have been effective include solutionfocused guided imagery (SFGI, a form of imagery therapy [15], cognitive behavior modification [3], botulinum toxin injections [16], and acupuncture [17].

Thus, studies on the yips have investigated the factors involved in its onset and the methods for coping with it. This context suggests that the yips is a difficult-to-treat condition and a danger for athletes, which brings with it the risk of them dropping out of competitions $[11,18]$. For example, Marquardt [19] reported that many athletes suffer from the yips and that those who have experienced the onset of the yips notice an exacerbation in their symptoms the more they try to escape the problem. Moreover, Milne and Morrison [20] and Smith, et al. [2] stated that the psychological anguish caused by the yips is very intense, and they referenced players losing their careers depending on the situation. As this has a huge impact on such athletes, the experience of the yips is highly detrimental in sports settings.

Clinical psychology and positive psychology studies have reported that negative experiences in life accompanied by a psychological struggle or conflict have an impact in the form of depression, anxiety disorders, and stress on individuals who 
experience them. However, these fields have also reported that such experiences can become an opportunity to achieve psychological growth [21]. For example, posttraumatic growth (PTG) is a representative concept that explains this psychological growth that accompanies these sorts of negative experiences [22]. PTG is defined as "the subjective experience of positive psychological change reported by an individual as result of the struggle with trauma" ([23], p. 628). Another concept that is known as widely as PTG is stress-related growth (SRG). LoSavio, et al.[24] reported that psychological growth can also be spurred by stressful experiences when there is confrontation in everyday life such as a conflict with a friend, family member, or significant other or in the case of poor performance at school or work.

Moreover, in recent years, experts in sport psychology have taken note of such concepts $[25,26]$. For example, Sarkar, et al [27] conducted semi-structured interviews on 10 Olympic gold medalists and had them describe their past adversity-related experiences (e.g., repeated non-selection, significant sporting failure, and serious injury). Many of the adversity-related experiences were considered a chance for personal psychological development as an athlete and as an impetus leading to success in the Olympics. Additionally, Sarkar, et al. [27] pointed out that based on these Olympic gold medalists' narratives, the athletes likely used these adversity-related experiences to further their ambitions as athletes who "want to be great" or "want to be better than anyone else." Furthermore, Stambulova [28] noted crises related to age, crises related to sporting career, and crises related to situations or the environment as the three primary crises experienced by athletes. This study suggests that these experiences of crises were turning points in athletes' psychological growth.

Thus, various studies have been conducted on the psychological growth that accompanies negative experiences in sports settings. Nevertheless, most of these previous studies have used qualitative approaches for their investigations [29] However, to measure the psychological growth of athletes, Sugiura[30] created a Psychological Maturity Scale for Athletes. This scale comprises the following five subscales: clear meaning of sports activity, self-understanding, independent achievement orientation, mental stability, and physiological controllability. This scale was used to examine quantitatively the relation between athletes' psychological growth and the various experiences of crises they face. The results indicated that going through experiences of crises in sports made people prone to having seen improvements in clear meaning of sports activity, self-understanding, and independent achievement orientation; however, mental stability and physiological controllability were not easily changed by these experiences. The former point is related to the desire to practice and the will to continue with competitions, while the latter is primarily related to exhibiting actual ability.

As mentioned above, there is considerable prior research demonstrating that the negative experiences of athletes have the potential to promote psychological growth. However, negative experiences of athletes increase their anxiety and heighten loss of self-confidence, and these experiences have negative aspects that can cause a decrease in motivation. On the other hand, research seems to suggest that the psychological struggles and conflicts that accompany such experiences have a dual nature whereby they also contain positive elements (e.g., being the impetus to achieve psychological growth). Considering this, the yips, which has only been viewed as a negative experience for athletes, can potentially become something that promotes athletes' psychological growth.

Studies have indicated that (1) the psychological growth that accompanies an individual's negative experiences takes place in the process of adapting to the problem faced, and that (2) the resolution of a crisis or the lessening of pain is not a necessary condition for their growth [31,32]. However, some reports also state that the experience of these sorts of events does not cause growth by itself, and that crisis resolution and the lessening of pain are necessary for growth $[33,34]$. Accordingly, in cases where there is some sort of psychological growth that accompanies athletes' experience of the yips, it is necessary to investigate whether it is essential to overcome the yips or if growth can occur without overcoming the yips.

Therefore, the present study aimed (1) to examine whether there is a relation between athletes' psychological growth and their experience of the yips and (2) to investigate if overcoming the yips plays a role in this psychological growth.

\section{Methods}

\section{Participants}

A target group comprising 457 players who were members of regulation baseball clubs at seven colleges were surveyed using three questionnaires and a face sheet profile (explained below). Forty-one people were excluded from the study because 26 of them returned inadequate responses to the questionnaire and the remaining 15 were deemed to fall outside the target group of this study for reasons mentioned below. Responses from 416 members $(\mathrm{M}=19.6, \mathrm{SD}=1.1$ year $)$ of university regulation baseball clubs were analyzed. The survey was conducted in a group setting at practice sessions or meetings. In all cases, the surveys were collected immediately after they were completed. Prior to the release of the survey, oral and written explanations of the purpose of the survey were provided, as were an overview of the yips, the relation of the yips to the present study, and how personal information would be managed. Those who seemed to have gained an adequate understanding of the given study and the yips and provided their consent to cooperate in the study were asked to respond to the questionnaire. As part of ethical considerations, those who were taking medication or commuting to hospitals for psychiatric conditions were excluded from the survey.

\section{Question items}

\section{Face sheet}

This profile contained questions on sex, years in college, years of experience competing, achievements in competition, main position, and whether the participant had the experiencing of being a captain. 


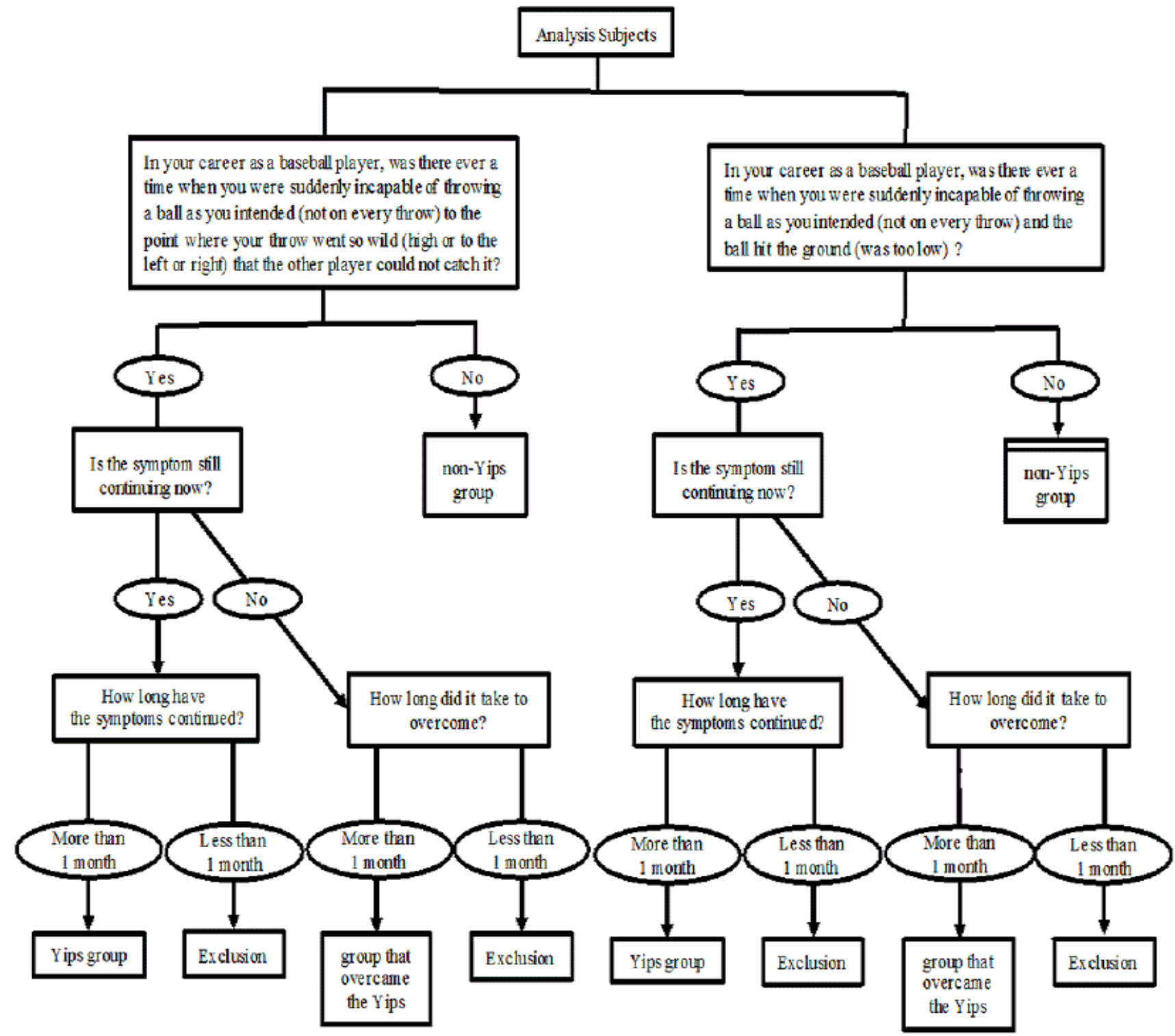

non-Yips group $(n=256)$

Yips group $(n=38)$

group that overcame the Yips $(n=69)$

Figure 1: Grouping of subjects (based on Uchida, 2008) 
Questionnaire on the yips: This questionnaire (Fig. 1) was created on the basis of a questionnaire used by Uchida [12]. First, the respondents answered "Yes" or "No" to the following two questions: "In your career as a baseball player, was there ever a time when you were suddenly incapable of throwing a ball as you intended (not on every throw) to the point where your throw went so wild (high or to the left or right) that the other player could not catch it?" and "In your career as a baseball player, was there ever a time when you were suddenly incapable of throwing a ball as you intended (not on every throw) and the ball hit the ground (was too low)?" After this question, the respondents answered questions about how long the yips lasted and how it could be improved.

\section{Yips Scale}

This scale was created by Uchida [12] to measure yips tendencies in baseball players who are part of high school and university baseball clubs. Five subscales within this scale were created from 21 items and given the following names: expectation anxiety, body image distortion, lack of a natural stance, advice from the environment, and affirmations from others. Expectation anxiety was assessed using six items such as "thinking that might be taken off the team if I threw wild." Body image distortion was assessed using six items such as "holding a ball, imagining a wild throw and feeling tension in the body." Lack of a natural stance was assessed using three items such as "not being able to throw like I want to when the distance is shorter than one base." Advice from the environment was assessed using three items such as "getting a lot of coaching and advice about throwing or pitching from the coaches." Affirmation from others was assessed using three items such as "why can other players throw where they want?" The responses were evaluated on 5-point scales ranging from "completely disagree" (1 point) to "completely agree" (5 points). Uchida [12] investigated this scale's validity using concurrent validity and criterion-related validity, and reliability was examined using Cronbach's alpha. Both validity and reliability had adequate values. Uchida [12] took the total scores of each of these factors as the yips score. Higher scores indicate stronger yips tendencies. Nevertheless, this scale assesses athletes' yips tendencies and cannot determine the onset of the initial symptoms of the yips. It is difficult to identify the yips using one scale alone. Therefore, the present study used this scale as a means of verifying the validity of the results of the aforementioned questionnaire on the yips.

\section{Psychological Maturity as an Athlete Scale}

This scale was created by Sugiura [30] to measure the extent of a person's psychological development as an athlete. The five subscales within this scale were developed from 32 items and named as follows: clear meaning of sports activity, self-understanding, independent achievement orientation, mental stability, and physiological controllability. The clear meaning of sports activity was assessed using seven items such as "I understand the reason why I am engaging in sports." Selfunderstanding was assessed using six items such as "I think I have an accurate grasp of my own abilities." Independent achievement orientation was assessed using six items such as "independently practicing without being told to practice by anyone else." Mental stability was assessed using six items such as "thinking optimistically about the results of a match." Physiological controllability was assessed using seven items such as "I know how to control my own movements." Responses were evaluated on 5-point scales ranging from "completely disagree" (1 point) to "completely agree" (5 points). Sugiura [30] investigated this scale's (1) content and criterion-related validity and (2) reliability using Cronbach's alpha. Both validity and reliability had adequate values.

\section{Analysis method}

All participants were classified based on the five evaluation criteria developed from prior research. The method for checking whether all these evaluation criteria were satisfied involved the face sheet and a questionnaire created as a partial revision of Uchida's questionnaire on the yips [12]. These five criteria were established based on common characteristics, the definition of the yips given above, and the evaluation criteria for the yips set out by Sachdev [35]. The first criterion was having at least five years of experience playing baseball [35]. The second was being acknowledged as having clear abnormalities in throwing or pitching motions [6]. The third was having a sudden inability to perform a motion that was previously very natural [2]. The fourth was being unable to throw or pitch a ball as intended[5]. Finally, the fifth was having symptoms that were not temporary and continued for at least one month [7,12]. The face sheet and the questionnaire on the yips were used to check whether these five criteria were satisfied. The participants were then categorized in the manner detailed below based on the questionnaire on the yips (Fig. 1). Further, the Yips Scale [12] was used to check the validity of the three groups into which the participants were classified. A one-way analysis of variance (ANOVA) was performed by (1) setting group (the non-yips group, yips group, and the group that overcame the yips) as the independent variable and (2) setting the five subscale scores in the Yips Scale as the dependent variable. A different one-way ANOVA was performed with group as the independent variable and the five subscale scores on the Psychological Maturity as an Athlete Scale as the dependent variables. This step sought to examine and clarify the relations between athletes' psychological development and the experience of the yips or overcoming the yips. A multiple-comparison test was subsequently performed using the Bonferroni correction where significant main effects were observed in the one-way ANOVA. For all tests, $p<.05$ was considered statistically significant.

\section{Results}

Table 1 shows the results of using the Yips Scale to verify the validity of the three groups into which the participants were divided based on the questionnaire on the yips. Upon examining the average values for each subscale score of the Yips Scale for the three groups using a one-way ANOVA, the following values were determined: expectation anxiety $(\mathrm{F}(2,414)=53.72, \mathrm{p}<.05$, $\eta 2=.16)$, body image distortion $(F(2,414)=105.99, \mathrm{p}<.05, \eta 2=$ $.34)$, lack of a natural stance $(F(2,414)=71.73, p<.05, \eta 2=.26)$, 
advice from the environment $(\mathrm{F}(2,414)=59.40, \mathrm{p}<.05, \eta 2=.22)$, and affirmation from others $(\mathrm{F}(2,414)=55.57, \mathrm{p}<.05, \eta 2=.21)$. Thus, because there was a significant main effect for all subscales, a multiple-comparison test was performed using the Bonferroni correction (Fig. 1). The results of this test indicated that the yips group ( $\mathrm{p}<.001)$ and the group that overcame the yips $(\mathrm{p}<.001)$ had significantly higher scores on all subscales as compared to the non-yips group. Further, the results revealed that the yips group had significantly higher scores on these subscales than did the group that overcame the yips $(\mathrm{p}<.001)$. The non-yips group had never experienced the yips, and hence, their score was the lowest. The group that had overcome the yips had the next lowest score, and the yips group, whose members were currently experiencing symptoms of the yips, had the highest yips score. These results verify the validity of classifying the subjects into the above-mentioned three groups.

Table1: descriptive statistics and analysis results on subscale score of the Yips Scale

\begin{tabular}{|c|c|c|c|c|c|}
\hline & $\begin{array}{l}\text { Non-Yips Group } \\
\text { (N = 256) } \\
M(S D)\end{array}$ & $\begin{array}{l}\text { Yips Group } \\
\text { (N = 38) } \\
M(S D)\end{array}$ & $\begin{array}{c}\text { Group That Overcame } \\
\text { The Yips }(\mathrm{N}=69) \\
M(S D)\end{array}$ & F-Value & $\begin{array}{c}\text { Multiple } \\
\text { Comparison }\end{array}$ \\
\hline Expectation Anxiety & $10.76(4.16)$ & $17.76(5.16)$ & $13.47(4.77)$ & $52.49^{*}$ & $2>3>1$ \\
\hline Body-Image Distortion & $7.11(2.77)$ & $14.38(4.31)$ & $9.41(3.67)$ & $105.54^{*}$ & $2>3>1$ \\
\hline Lack Of A Natural Stance & $4.18(1.77)$ & $7.86(2.29)$ & $5.67(2.57)$ & $71.73^{*}$ & $2>3>1$ \\
\hline Advice From The Environment & $4.89(2.14)$ & $8.64(2.30)$ & $6.41(2.54)$ & $59.4^{*}$ & $2>3>1$ \\
\hline Affirmations From Others & $6.31(2.49)$ & $10.36(1.82)$ & $8.15(3.06)$ & $55.57^{*}$ & $2>3>1$ \\
\hline
\end{tabular}

Table2: descriptive statistics and analysis results on subscale score of the psychological maturity as an athlete

\begin{tabular}{|c|c|c|c|c|c|}
\hline & $\begin{array}{c}\text { Non-Yips Group } \\
\text { (N = 256) } \\
M(S D)\end{array}$ & $\begin{array}{l}\text { Yips Group } \\
\text { (N = 38) } \\
M(S D)\end{array}$ & $\begin{array}{c}\text { Group That } \\
\text { Overcame The } \\
\text { Yips (N = 69) } \\
M(S D)\end{array}$ & F-Value & $\begin{array}{c}\text { Multiple } \\
\text { Comparison }\end{array}$ \\
\hline clear meaning of sports activity & $28.29(5.57)$ & $26.71(5.76)$ & $29.97(4.47)$ & $5.27^{* *}$ & $3>1,2$ \\
\hline self-understanding & $22.43(4.40)$ & $22.40(3.88)$ & $24.2(3.76)$ & $5.94^{* *}$ & $3>1$ \\
\hline independent achievement orientation & $21.18(4.86)$ & $20.83(4.45)$ & $22.65(4.11)$ & $3.31^{*}$ & $3>1$ \\
\hline mental stability & $17.32(4.73)$ & $15.38(4.63)$ & $16.76(4.31)$ & $3.34^{*}$ & $1>2$ \\
\hline physiological controllability & $22.22(5.37)$ & $17.57(4.93)$ & $20.84(5.02)$ & $15.07^{* * *}$ & $1,3>2$ \\
\hline
\end{tabular}

${ }^{*} \mathrm{p}<.05,{ }^{* *} \mathrm{p}<.01^{* * *}, \mathrm{p}<.001$ multiple comparison $1=$ non-Yips group; 2 =Yips group; $3=$ group that overcame the Yips

Table 2 shows the average values for the subscale scores of the Psychological Maturity as an Athlete Scale for each of the three groups (the non-yips group, the yips group, and the group that overcame the yips). Upon examining the average values for each subscale score of the Psychological Maturity as an Athlete Scale for the three groups using a one-way ANOVA, the following values were determined: clear meaning of sports activity $(\mathrm{F}(2,413)=$ 5.27, $\mathrm{p}<.05, \mathrm{\eta} 2=.03)$, self-understanding $(\mathrm{F}(2,413)=5.95, \mathrm{p}<$ $.05, \eta 2=.03)$, independent achievement orientation $(\mathrm{F}(2,413)=$ $3.31, \mathrm{p}<.05, \eta 2=.02)$, mental stability $(\mathrm{F}(2,413)=3.36, \mathrm{p}<.05$, $\eta 2=.02)$, and physiological controllability $(\mathrm{F}(2,413)=15.07, \mathrm{p}<$ $.05, \eta 2=.07)$. Thus, as there was a significant main effect for all subscales, a multiple-comparison test was performed using the Bonferroni correction (Fig. 1). There were significant differences between the group that overcame the yips and the non-yips group $(p=.049)$ and between the group that overcame the yips and the yips group $(p=.006)$ in their clear meaning of sports activity scores. In both cases, the group that overcame the yips had higher scores. Additionally, the group that overcame the yips had higher scores than did the non-yips group for self-understanding 
$(\mathrm{p}=.006)$ and independent achievement orientation $(\mathrm{p}=.047)$ Moreover, the non-yips group had significantly higher scores on mental stability than did the yips group $(p=.035)$. Finally, there was a significant difference in the physiological controllability score between the non-yips group and the yips group $(\mathrm{p}<.001)$ and between the group that overcame the yips and the yips group $(\mathrm{p}=.004)$. In both cases, the yips group had lower scores.

\section{Discussion}

This study divided 416 baseball players into three groups: a non-yips group, a yips group, and a group that overcame the yips. Next, it clarified the difference between the groups' psychological development scores to examine the relation between baseball players' degree of psychological development and their experience of the yips and overcoming the yips.

\section{Clear meaning of sports activity}

The group that overcame the yips had higher scores for the clear meaning of sports activity than did the yips and non-yips groups. These results seem to indicate that overcoming the yips contributes greatly to increased scores on the clear meaning of sports activity. People who have experienced the yips have the following experiences while the symptoms of the yips persist-a stirring up of negative emotions such as "feeling disappointed or frustrated" [36] or a "loss of confidence" [36]. While experiencing symptoms of the yips, difficulties were encountered due to things like being unable to practice like everyone else or play matches.

During this period, these players considered questions such as "Why do I play sports?" or "Is it necessary for me personally to continue with sports?" As shown by the results of the present study, while people are experiencing the yips, they are naturally prone to reduced scores on the clear meaning of sports activity or are in a state where it is difficult to provide support on the clear meaning of sports.

However, Sugiura[37] assumed that an athlete's crisis about their reason for participating in sports or a crisis in motivation for participation in sports "makes athletes think about why they are playing sports and what type of athlete they are"([37], p. 191). Moreover, Sugiyama proposed that resolving a crisis in the motivation for participation in sports could lead to psychological development as an athlete. If those findings and the results of the present study are considered jointly, one can conclude that people who have experienced the yips think deeply about their own reason for continuing baseball in the process of handling the psychological conflict that accompanies the experience, and use overcoming the yips as the impetus to find a new purpose or develop a clearer purpose than they had before experiencing the yips.

\section{Self-understanding}

The group that overcame the yips had significantly higher scores on self-understanding than did the non-yips group, suggesting that those who have experienced the yips and overcome it have a more accurate understanding of their own abilities, physical condition, attitude, and how they should practice compared with those who have never experienced the yips.

Tamminen, et al. [26] conducted semi-structured interviews on elite athletes to study the negative experiences of athletes in sports settings or their daily lives and the accompanying psychological development of the athletes. The results of this study show that some players gain a strong understanding of the limitations of their own abilities and the strength of their body and mind through their negative experiences, and some players gained an understanding of their own identity beyond their identity as an athlete. Additionally, Wadey, et al. [38] examined the psychological transformation of injured athletes in the process of recovering from their injuries, and found that in their struggle to recover from injuries or in the psychological conflict that they face, injured athletes reflect on themselves, try to understand their own emotions, and actively disclose information about their own emotions to other people. It is possible that through these processes, these athletes come to have a strong understanding of their own emotions. In addition, it was reported that athletes' negative experiences result in positive transformations such as gaining a strong understanding of their own abilities and what they can and cannot do [38, 39]spurring personal realizations and acquisition of insight [40].

There have been many reports of how negative experiences in everyday life or sports settings can present a good opportunity for self-reflection, allowing a deeper understanding of one's own abilities, circumstances, and identity. It seems clear that the experience of the yips is a chance for baseball players to reflect on themselves; moreover, it seems that overcoming the yips is an opportunity to reach a deeper understanding of athletes' own abilities and themselves.

\section{Independent achievement orientation}

The group that overcame the yips had significantly higher scores on independent achievement orientation than dud the non-yips group. In other words, those who have experienced and overcome the yips had a more active attitude toward practice and competition than did those who have not experienced the yips.

Nakamura and Araki [41] investigated the process of injured athletes accepting their injury or recovering from it. Their study clarified that by going through the process of coping with a sports injury, people reported positive transformations such as experiencing joy in being able to compete or having a positive attitude. Additionally, Podlog and Eklund [42] conducted a longitudinal study investigating the transformation that takes place in the recovery of competitive athletes who suffer a serious injury. Most participants reconsidered the importance of sports in their lives and the value of being able to play sports because they could not play sports the way they wanted to due to an injury. This increased their motivation to participate in sports and their desire to want to play sports. The results of negative experiences in sports help athletes obtain a renewed sense of joy, gratitude, or happiness for being able to play sports. People who have experienced the yips have painful experiences, such as being transferred to a different position [7], not being able to 
play in matches [16], and becoming unable to execute behaviors that were previously simple and natural [8]. Therefore, those who have experience with and have overcome the yips truly enjoy daily practice and value the ability to compete in a way that they had not felt or had forgotten before their experience with the yips. These people actively compete or train because of these experiences.

\section{Mental stability}

The yips group had significantly lower scores on mental stability compared with the non-yips group. People who have experienced the yips may continue to compete while constantly struggling with psychological tension, feelings of anxiety that yips symptoms might appear in front of spectators, and fixations on other people's assessments [9], leading them to become psychologically unstable.

In recent years, however, many studies have reported positive transformations with regard to mental stability that takes place through these negative experiences in a sports setting. These transformations include increases in mental toughness [42] and resilience and decreases in performance anxiety [43], as well as improved coping skills[38,44,45]. Accordingly, further research is required.

\section{Physiological controllability}

A significant difference was seen in the physiological controllability score between the non-yips group and the yips group and between the group that overcame the yips and the yips group; in both cases, the yips group had lower scores. This suggests that physiological or psychological symptoms shared by people who have experienced the yips may play a role in the yips group. These physiological symptoms of the yips include muscular dysfunction such as muscle testing, jerking, twitching, tremors, or freezing, observed in performance of the action where the yips appear $[2,46]$. Further, the main psychological symptoms that occurred were a sense of being unable to deftly control one's own body $[6,36]$, and some had specific complaints such as "Too much tension in my fingers," "It felt as if my shoulder had locked up," and "I could not let go of the ball." This seems to suggest that those who have experienced the Yips may lose their selfconfidence, particularly about being able to deftly control their own bodies, while experiencing symptoms of the yips. This could be why the yips group showed markedly lower physiological controllability scores than did the non-yips group.

The group that had overcome the yips had significantly higher scores on physiological controllability, suggesting that these physiological or psychological issues related to the body improved when the yips were overcome. The athletes gradually regained self-confidence, becoming able to relax and execute proper performance.

Are the significantly higher scores (in comparison to the yips group) of the non-yips group and the group that overcame the yips of the same quality? The main symptom of the yips is losing control of a specific part of the body. Therefore, even if symptoms improve in athletes who have experienced the loss of such control, it is difficult to believe that their sense of their body and self-confidence about controlling their body could return to as it was before they experienced the yips. In short, it seems possible that the non-yips group members and the members the group that overcame the yips would have different characteristics in terms of psychological development. Accordingly, there is still room for further study on physiological controllability.

Sugiura [30] reported that experiences of crises in sports tend to make it easier to see improvements in the clear meaning of sports activity, self-understanding, and independent achievement orientation. However, these experiences make improvements in mental stability and physiological controllability more difficult. Thus, the results of the present study support Sugiura [30]. Because the group that overcame the Yips had significantly higher scores on clear meaning of sports activity, self-understanding, and independent achievement orientation compared with the non-yips group, the experience of the yips and overcoming it may have triggered psychological development. On the other hand, the study did not show a higher psychological development score for the yips group or the group that overcame the yips for mental stability or physiological controllability compared with the non-yips group. This suggests that there is a tendency for the experience of the yips and overcoming it to act as a trigger to make it easy to improve on the former three subscales; however, it tends to be difficult to improve on the latter two. Accordingly, it may be that experiencing and overcoming the Yips mainly promotes the desire to practice and encourages people to participate in competitions rather than spurring psychological development related to the demonstration of ability.

Lastly, a noteworthy finding was that the subscales on which the group that had overcome the yips showed significantly higher scores than did the non-yips group were clear meaning of sports activity, self-understanding, and independent achievement orientation. Contrastively, there were no subscales where the Yips group had significantly higher scores than did the nonyips group. This result seems to substantiate the findings of Kamijyo and Yukawa [33] and Taubman-Ben-Ari, et al. [34], who noted that personal growth as a result of negative experiences does not happen simply because of the experience but because of the important roles played by resolution of the crisis or alleviation of the pain of negative experiences. In other words, the psychological development observed in people who have experienced the yips is not merely because of the experience of the yips, as overcoming the yips also plays an important role in this development. Accordingly, making effort and not evading painful experiences and the increased self-confidence resulting from being able to overcome an issue [47] affects psychological development. Further, Sugiura[30] noted the possibility that athletes gain a clear understanding of sports activities, a clear self-understanding, and an independent achievement orientation by overcoming performance-related problems or frequent slumps. Considering these findings and the results of the present study, it is understandable that only the group that has overcome the yips had higher scores than did the non-yips group. 


\section{Limitation and Future research}

This study used the scale proposed by Sugiura [30]; however, it is possible that people who have experienced the Yips undergo positive changes in areas other than thepsychological development measured on the scale. For example, Wadey, et al. [38] conducted interviews on the perceived benefits following sports injuries and reported that some injured athletes undergo a change in the process of coping with a sports injury where they become more altruistic, more considerate of the people around them, and begin to empathize with other injured athletes on their team. Per this report, psychological development has characteristics that have been observed in the studies on psychological development that accompanies negative experiences in sports settings, which are not found on the scales used [30].

This was a cross-sectional study, and hence, we cannot establish a causal relationship. Therefore, while the group that overcame the yips was observed to have significantly higher scores on the three subscales clear meaning of sports activity, self-understanding, and independent achievement orientation compared with the non-yips group, it is not clear whether this difference really came from experiencing and overcoming the yips.

Accordingly,more detailed investigations are necessary in the future. These investigations should conduct surveys of individual baseball players who have experienced the yips with questions such as "Did you experience any type of psychological development that was not included in the Psychological Maturity as an Athlete Scale?"or "Did experiencing or overcoming the yips really trigger you to experience psychological development as an athlete?"Additionally,the advantages of using this type of qualitative approach is that it is possible to explore in depth the characteristics of the psychological transformations among people who have persisting symptoms of the yips (the yips group). The results of this study did not indicate a significant difference between the non-yips group and the yips group,but it is possible that people with persistent symptoms of the yips (the yips group) experience psychological development that cannot be measured using existing scales,due to a mixture of ambivalent feelings about psychological struggle that accompanied the experience of the yips. Sugiura [30] used (1)a quantitative survey that employed the Psychological Maturity as an Athlete Scale and (2)a qualitative survey on the same target group to clarify the relation between psychological development and the experiences of crisis as an athlete. That survey reported the characteristics of psychological development that were not measurable in the survey,observed in the results of the free descriptions. Additionally,Hiraki [48] reported that in the interviews done regarding PTG,there were signs of growth in people who were still suffering the pain of not being able to solve their problem.

The next limitation related to the target group of the survey is that the selection of the target group in the present study was only based on the results of subjective responses given by respondents to a questionnaire. However, whether these respondents had truly experienced the yips or had overcome it could not be verified objectively. Thus, when a target group for a survey is selected in the future, responses should also be taken from the players' coaches or teammates. Alternatively, conducting objective tests on physiological, praxiological, or neurological aspects might enable a more accurate measurement of the relation between experiencing or overcoming the yips and psychological development. Finally, while the present study might be seen as glorifying the experience of the yips, it does not claim that the experience of the yips is necessary for psychological development as an athlete. Additionally, the results of this study do not show whether anyone can achieve psychological development even if they have experienced the yips. Moreover, even if one actually could achieve psychological development triggered by the experience of the yips, it is possible that it could lead to negative psychological transformations, such as increased expectation anxiety, decreased motivation, or a loss of selfconfidence (e.g., [36]). Baker, et al.'s study on PTG [49] reported that there are both positive and negative aspects in the process of a psychological transformation that accompanies negative experiences in life. Thus, it is possible that the experience of the yips would trigger psychological development in athletes; however, it is also essential to understand that it could also produce negative effects.

\section{References}

1. McDaniel KD, Cummings JL, Shain S. The "yips" a focal dystonia of golfers. Neurology. 1989;39(2):192-195.

2. Smith AM, Malo SA, Laskowski ER, Sabick M, Cooney WP 3rd, Finnie $\mathrm{SB}$, et al. A multidisciplinery study of the "yips" phenome- non in golf: an exploratory analysis. Sports medicine. 2000;30(6):423-437.

3. Kagawa M. Effect of mental training with self-monitoring and cognitive restructuring by video feedback for a college baseball player with yips symptom. Mental training journal. 2013;7:35-44.

4. Weiss SM, Reber AS. Curing the dreaded "Steve Blass disease". Journal of sport psychology in action. 2012;3(3):171-181. Doi: $10.1080 / 21520704.2012 .682702$

5. Nakagomi S. How to cope with the embodied mental health problems, "The yips", Special topic: how to identify and cope with when the mind in weak-Early response rescues players. Training journal. 2006;28(2):30-34.

6. Bawden M, Maynard I. Towards an understanding of the personal experience of the 'yips' in cricketers. Journal of sports sciences. 2001;19(12):937-953. Doi: 10.1080/026404101317108444

7. Martin G. The yips continuum: The psychology of sports performance break-downs (Doctor dissertation, Chicago school of professional psychology). 2015.

8. Mukai K. Study of the psychological process during the yips in baseball experience using the traje- ctoryequifinality model. Japanese journal of qualitative psychology. 2016;15:159-170.

9. Iwata I. Hasegawa K. An approach from the standpoint of sports clinical psychology to psychogenic motordisorder. Japanese society of sport psychology. 1981; 8(1):28-34.

10. Nakagomi S. A Psychotherapeutic look at a baseball pitcher's difficulty in throwing the ball at a certain distance: the psychological distance of interpersonal relationships projected into the distance of throwing. Japanese society of sport psychology. 1987;14(1): 58-62. 
11. Kagawa M, Fukae M. A study on psychological trait of junior high school baseball player with symptom of throwing and catching ataxia. Research bulletin of narutouniversity of education. 2013;28:440-453.

12. Uchida, M. Development of the yips scale in baseball (master's thesis, Juntendo university graduate school of health and sports science).2008.

13. Adler CH, Crews D, Kahol K, Santello M, Noble B, Hentz JG, et al. Are the yips a task-specific dystonia or "golfer's cramp"?.Movement disorders. 2011;26(11):1993-1996. Doi: 10.1002/mds.23824.

14. Klämpfl MK, Lobinger BH, Raab M. Reinvestment-the cause of the yips?.PloSone. 2013;8(12);e82470.Doi: 10.1371/journal. pone. 0082470

15. Bell RJ, Thompson CL. Solution-focused guided imagery for a golfer experiencing the yips: a case study. Athletic insight. 2007;9(1):52-66.

16. Dhungana S, Jankovic J. Yips and other movement disorders in golfers. Movement Disorders. 2013;28(5):576-581.Doi: 10.1002/ mds.25442

17. Rosted P. Acupuncture for treatment of the yips?-A case report. Acupuncture in medicine. 2006;23(4):188-189.

18. Bell RJ, Skinner CH, Fisher LA. Decreasing putting yips in accomplished golfers via solution-focused guided imagery: A single-subject research design. Journal of Applied Sport Psychology.2009;21(1):1-14.Doi: $10.1080 / 10413200802443776$

19. Marquardt $\mathrm{C}$. The vicious circle involved in the development of the yips. International journal of sports science \& coaching. 2009;4(1_ suppl):67-88.

20. Milne D, Morrison G. Cognitive behavioural intervention for the golf yips: A singlecase design. sport \& exercise psychology review. 2015;11(1):20-33

21. Lechner SC, Carver CS, Antoni MH, Weaver KE, Phillips KM. Curvilinear associations between benefit finding and psychosocial adjustment to breast cancer. Journal of consulting and clinical psychology, 2006;74(5):828-840.10.1037/0022-006X.74.5.828

22. Tedeschi RG, Calhoun LG. The posttraumatic growth inventory: measuring the positive legacy of trauma. Journal of traumatic stress. 1996;9(3):455-471. 10.1002/jts.2490090305

23. Zoellner T, Maercker A. Posttraumatic growth in clinical psychology-A critical review and introduction of a two component model. Clinical psychology review. 2006;26(5):626653. Doi: $10.1016 /$ j.cpr.2006.01.008

24. LoSavio ST, Cohen LH, Laurenceau JP, Dasc KB, Parrish BP, Park CL. Reports of stress-related growth from daily negative events. Journal of social and clinical psychology.2011:30(7);760-785.Doi; 10.1521/ jscp.2011.30.7.760

25. Salim J, Wadey R, Diss C. Examining hardiness, coping and stressrelated growth following sport injury. Journal of applied sport psychology. 2016;28(2):154-169. Doi:10.1080/10413200.2015.108 6448

26. Tamminen KA, Holt NL, Neely KC. Exploring adversity and the potential for growth among elite female athletes. Psychology of sport and exercise. 2013;14(1):28-36. Doi:10.1016/j.psychsport.2012.07.002

27. Sarkar M, Fletcher D, Brown DJ. What doesn't kill me...: adversityrelated experiences are vital in the development of superior Olympic performance. Journal of scienceand medicine in sport.

\section{5;18(4):475-479. Doi: 10.1016/j.jsams.2014.06.010}

28. Stambulova NB. Athlete's crises: A developmental perspective. International journal of sport psychology. 2000;31:584-601.

29. Tamminen KA, Neely KC. Positive growth in sport. In N. L. Holt (ed.), Positive youth development through sport. 2nd ed. London: Routledge. 2016; p. 193-204.

30. Sugiura T. The empirical study of mental maturity as an athlete. Japan journal of physical education, health and sport sciences. 2001;46:337-351.

31. Park CL, Cohen LH, Murch RL. Assessment and prediction of stressrelated growth. Journal of personality. 1996; 64(1):71-105.

32. Kimura S, Oishi K. An analysis of japanese college athletes' views regarding extracurricular sports activities using a quantitative text analysis: Coping strategies and psychological changes. Journal of the institute of community \& human services, Rikkyo university. 2016;4:17-33.

33. Kamijyo N, Yukawa S. Characteristics of stressful events and motivation to make meaning: A basic study using hypothetical scenarios. Japanese journal of counseling science. 2014;47(3):137146.

34. Taubman-Ben-Ari O, Findler L, Shlomo SB. Personal growth and the transition to grandfather-hood. The international journal of aging and human development. 2012;74(4):265-285.

35. Sachdev P. Golfers' cramp: clinical characteristics and evidence against it being an anxiety disorder. Movement disorders. 1992;7(4):326332.Doi: 10.1002/mds.870070405

36. Philippen PB, Lobinger B H. Understanding the yips in golf: thoughts, feelings, and focus of attention in yips-affected golfers. Sport psychologist. 2012; 26(3): 325-340

37. Sugiura T. Cosnstructing a theory of psychological maturity as athlete. Kyoto university research studies in education. 1996;(42):188-198.

38. Wadey R, Evans L, Evans K, Mitchell I. Perceived benefits following sport injury: Aqualitative examination of their antecedents and underlying mechanisms. Journal of applied sport psychology. 2011;23(2):142-158.Doi: 10.1080/10413200.2010.543119

39. Udry E, Gould D, Bridges D, Beck L. Down but not out: Athlete responses to season ending injuries. Journal of sport and exercise psychology. 1997;19(3):229-248.Doi: 10.1123/jsep.19.3.229

40. Yonemaru K, Suzuki M. Psychological counseling for an athlete who complained "I'm not sure what I am"-The process of growing up to become an independent athlete through dialogue-. Japanese journal of sport psychology. 2016;43(1):15-28.Doi: 10.4146/ jjspopsy.2015-1508

41. Nakamura T, Araki M. The application of "The five stage of grief" to acceptance of athletic injury: Case studies with qualitative approach. Osaka society of physical education. 2016;54:31-40.

42. Podlog L, Eklund RC. A longitudinal investigation of competitive athletes' return to sport following serious injury. Journal of applied sport psychology. 2006;18(1):44-68.

43. Kobayashi Y, Yamaguchi K, Matsuda M, Hashimoto S. Health28-014 About psychological factors related to sports injuries of Judo players and potential of psychological growth. 10 Health, General announcement abstract. Japan society of physical education, health and sport sciences conference proceedings. 2014:65;286-287. 
44. Galli N, Reel JJ. 'It was hard, but it was good': a qualitative exploration of stress-related growth in Division I intercollegiate athletes. Qualitative research in sport, exercise and fealth. 2012;4(3):297-319. Doi: 10.1080/2159676X.2012.693524

45. Wadey R, Clark S, Podlog L, McCullough D. Coaches' perceptions of athletes' stress-related growth following sport injury. Psychology of Sport and Exercise. 2013;14(2):125-135.Doi: 10.1016/j. psychsport.2012.08.004

46. Smith AM, Adler CH, Crews D, Wharen RE, Laskowski ER, Barnes K, Valone Bell C, Pelz D, Brennan RD, Smith J, Sorenson MC, Kaufman KR. et al. The 'yips' in golf: a continuum between a focal dystonia and choking. Sports medicine. 2003;33:13-31.
47. Matsushita T. The changing process about ways of finding meaning in negative experience: Taking notice of positive meaning (clinical psychology). Kyushu university psychological research. 2008;9:101110.

48. Hiraki K. Chapter 2Accompany a journey of growth-What I learned from the narrative of survivor. In Taku, K. (Ed.), potential and challenge of PTG. Tokyo: Kaneko-shobo;2016.p.22-34.

49. Baker JM, Kelly C, Calhoun LG, Cann A, Tedeschi RG. An examination of posttraumatic growth and posttraumatic depreciation: Two exploratory studies. Journal of Loss and Trauma. 2008;13(5):450465. Doi: $10.1080 / 15325020802171367$ 